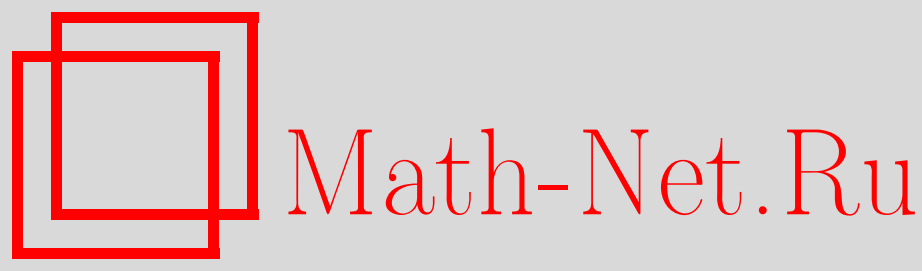

А. А. Шейкин, С. А. Пастон, Классификация минимальных глобальных вложений для невращающихся черных дыр, ТМФ, 2015, том 185, номер 1, 213-223

DOI: https://doi.org/10.4213/tmf8920

Использование Общероссийского математического портала Math-Net.Ru подразумевает, что вы прочитали и согласны с пользовательским соглашением http://www . mathnet.ru/rus/agreement

Параметры загрузки:

IP : 3.81 .55 .215

26 апреля 2023 г., 13:35:12

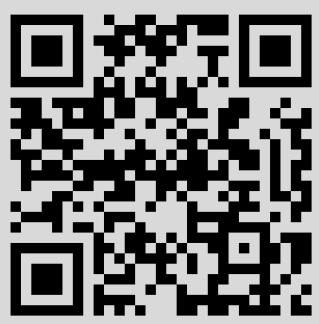




\title{
КЛАССИФИКАЦИЯ МИНИМАЛЬНЫХ ГЛОБАЛЬНЫХ ВЛОЖЕНИЙ ДЛЯ НЕВРАЩАЮЩИХСЯ ЧЕРНЫХ ДЫР
}

\begin{abstract}
Рассматривается проблема существования глобальных вложений метрик сферически-симметричных черных дыр в объемлющее пространство минимальной возможной размерности. Возможные типы вложений классифицируются по типу реализации симметрии метрики симметриями объемлющего пространства. Для черных дыр Шварцшильда, Шварцшильда-де Ситтера и РейснераНордстрёма доказано отсутствие каких-либо глобальных вложений, кроме уже известных. Для метрики Рейснера-Нордстрёма-де Ситтера получено новое глобальное вложение, а для метрики Шварцшильда-анти-де Ситтера доказана невозможность построения искомых вложений. Также обсуждается возможность построения глобальных вложений метрики Рейснера-Нордстрёма-антиде Ситтера.
\end{abstract}

Ключевые слова: теория гравитации, изометрическое вложение, теория вложения, черные дыры, дополнительные измерения.

DOI: $10.4213 / \operatorname{tmf} 8920$

\section{1. ВВЕДЕНИЕ}

Согласно теореме Жане-Картана-Фридмана [1] произвольное $n$-мерное псевдориманово многообразие всегда можно локально изометрически вложить в плоское пространство размерности $N \geqslant n(n+1) / 2$. Для некоторых случаев, чаще всего при наличии какой-либо симметрии, достаточное значение $N$ может оказаться и меньше [2], а вложение может быть не только локальным, но и глобальным. Под вложением понимается нахождение такой поверхности в плоском объемлющем пространстве, описываемой функцией вложения $y^{a}\left(x^{\mu}\right)$, индуцированная метрика которой

$$
g_{\mu \nu}=\left(\partial_{\mu} y^{a}\right)\left(\partial_{\nu} y^{b}\right) \eta_{a b}
$$

воспроизводит заданную метрику вкладываемого псевдориманова многообразия (здесь и далее индексы $\mu, \nu, \ldots$ нумеруют его координаты, а индексы $a, b, \ldots$ - лоренцевы координаты объемлющего пространства; величина $\eta_{a b}$ - плоская метрика объемлющего пространства).

${ }^{*}$ Санкт-Петербургский государственный университет, Санкт-Петербург, Россия. E-mail: anton.shejkin@gmail.com,paston@pobox.spbu.ru 
Поскольку в рамках общей теории относительности наше пространство-время представляет собой псевдориманово многообразие, для него можно совершить процедуру вложения и описывать его как четырехмерную поверхность в плоском объемлющем пространстве. Его размерность $N$ в общем случае достаточно брать равной 10, а при вложении конкретных симметричных метрик она обычно оказывается меньше (отметим, однако, что в некоторых подходах используют и бо́льшие значения, например $N=14$ [3]). Вложения с наименьшей для данной метрики размерностью объемлющего пространства $N_{\min }$ называются минимальными.

Вложения начали применяться в общей теории относительности почти сразу после ее появления. В частности, первое вложение метрики Шварцшильда было построено в 1921 г. [4], спустя всего пять лет после ее нахождения. Использовать вложения в общей теории относительности можно с разными целями. Вплоть до семидесятых годов прошлого века вложения применялись главным образом для классификации точных решений [2], поскольку класс вложения $p=N_{\min }-n$ является инвариантом, а также для изучения геометрических свойств метрик - к примеру, известные координаты Крускала для метрики Шварцшильда были, скорее всего, получены с использованием вложения Фронсдала [5]. В работе [6] была впервые предложена идея использовать функцию вложения $y^{a}(x)$ вместо метрики $g_{\mu \nu}(x)$ в качестве динамической переменной, описывающей гравитацию. Различные варианты такого описания гравитации как теории вложения впоследствии исследовались неоднократно (см., например, работы [7]-[15]), иногда возникая независимо.

Явные вложения физически интересных решений уравнений Эйнштейна могут быть полезны не только для анализа структуры возникающего многообразия. Существуют попытки установить связь между квантовыми эффектами, возникающими в римановом пространстве и в плоском объемлющем пространстве на вложенной поверхности. Например, обсуждается соответствие между эффектами Хокинга и Унру при рассмотрении вложений [16] (см. также работы [17], [18] и цитируемую в них литературу). В качестве недавнего примера такого использования явных вложений можно упомянуть работу [19], где с помощью вложений вычисляется функция Грина в римановом пространстве.

Отметим, что сравнительно легко поддаются исследованию метрики с $p \leqslant 2$. Поскольку все сферически-симметричные черные дыры имеют класс вложения 2 (следуя [20], можно проверить, что для них $p \neq 1$, а для сферически-симметричной метрики общего вида $p=2$ ), в настоящее время для них известно достаточно много вложений. Вложения метрики Шварцшильда в шестимерное пространство, кроме уже упоминавшихся работ [4], [5], были предложены также в работах [21]-[23], причем в работе [23] были найдены все возможные минимальные вложения, допускаемые симметрией метрики. Из шести возникающих вложений четыре оказываются глобальными, т. е. гладкими при всех значениях радиуса, а два других (предложенные в работах [4], [21]) не могут быть продолжены под горизонт.

Для метрики заряженной черной дыры Рейснера-Нордстрёма минимальные вложения впервые были предложены в работах [24], [25], однако в наиболее интересном случае наличия двух горизонтов (так называемый неэкстремальный случай) они не являются глобальными. Полный набор симметричных глобальных минимальных вложений для неэкстремального случая был найден в работе [26]. 
Кроме уже упомянутых, среди сферически-симметричных еще известны незаряженные и заряженные черные дыры в пространствах де Ситтера и анти-де Ситтера (для незаряженных мы будем использовать обозначения S-dS и S-AdS соответственно, а для заряженных - RN-dS и RN-AdS). Для них есть только отрывочная информация о существовании глобальных вложений (неглобальные же легко получаются простым обобщением известных вложений метрики Шварцшильда). Например, в работе [27] предложены семимерные (следовательно, не минимальные) глобальные вложения для S-AdS и RN-AdS черных дыр. Минимальные глобальные вложения для S-dS черной дыры были найдены в работе [28]. Вложения черных дыр, не обладающих сферической симметрией, т. е. вращающихся черных дыр Керра и Керра-Ньюмена, практически не изучены. Существуют только девятимерные вложения Кузеева [29], [30] для метрик Керра и Керра-Ньюмена соответственно, 14-мерное вложение метрики Керра, предложенное в работе [31], а также различные диаграммы вложения (вложения подмногообразий, например, с $\phi=0$ ), которыми в этой работе мы интересоваться не будем.

В настоящей работе мы исследуем вопрос о существовании глобальных минимальных (т. е. шестимерных) вложений всех невращающихся черных дыр, рассматривая неэкстремальный случай, соответствующий максимально возможному числу горизонтов.

\section{2. ИССЛЕДУЕМЫЕ МЕТРИКИ И ТИПЫ ВЛОЖЕНИЙ}

Произвольную сферически-симметричную метрику, инвариантную относительно сдвигов параметра $t$ (т. е. статическую в областях с обычными свойствами причинности, в которых приращение $t$ соответствует времениподобному интервалу), можно записать в виде

$$
d s^{2}=g_{00}(r) d t^{2}+g_{11}(r) d r^{2}-r^{2}\left(d \theta^{2}+\sin ^{2} \theta d \varphi^{2}\right) .
$$

Общему случаю невращающейся черной дыры соответствуют компоненты метрики

$$
g_{00}(r)=1-\frac{2 m}{r}+\frac{q^{2}}{r^{2}}-\frac{\Lambda r^{2}}{3} \equiv F(r), \quad g_{11}(r)=-\frac{1}{F(r)},
$$

где $m>0$ - масса черной дыры, $q$ - ее заряд, а $\Lambda$ - космологическая постоянная, положительная, если рассматривается черная дыра в пространстве де Ситтера, и отрицательная - если в пространстве анти-де Ситтера.

Будем исследовать вложения для следующих шести типов невращающихся черных дыр:

1) Шварцшильда (S),

2) Шварцшильда-де Ситтера (S-dS),

3) Шварцшильда-анти-де Ситтера (S-AdS),

4) Рейснера-Нордстрёма (RN),

5) Рейснера-Нордстрёма-де Ситтера (RN-dS),

6) Рейснера-Нордстрёма-анти-де Ситтера (RN-AdS).

Ограничимся изучением таких областей значений параметров $m, q$ и $\Lambda$, при которых черная дыра не является экстремальной, т. е. имеет максимально возможное число горизонтов. Это соответствует случаю, когда заряд $q$ и космологическая 
ТАБлицА 1. Значения параметров $q, \Lambda$ и $n_{\text {hor }}$ для рассматриваемых черных дыр.

\begin{tabular}{|c|c|c|c|c|c|c|}
\hline & S & S-dS & S-AdS & RN & RN-dS & RN-AdS \\
\hline$q$ & 0 & 0 & 0 & $\neq 0$ & $\neq 0$ & $\neq 0$ \\
\hline$\Lambda$ & 0 & $>0$ & $<0$ & 0 & $>0$ & $<0$ \\
\hline$n_{\text {hor }}$ & 1 & 2 & 1 & 2 & 3 & 2 \\
\hline
\end{tabular}

постоянная $\Lambda$ достаточно малы. Возможные значения параметров $q$ и $\Lambda$, а также числа горизонтов $n_{\text {hor }}$ для таких черных дыр приведены в табл. 1 . Графики функций $g_{00}(r)$ для рассматриваемых черных дыр показаны на рис. 1.

В работе [23] была проведена классификация всех шестимерных вложений (т. е. четырехмерных поверхностей в плоском шестимерном пространстве), обладающих симметрией невращающихся черных дыр - симметрией $S O(3) \times T^{1}$. Выпишем возникающие девять типов вложений, указывая для каждого типа допустимые сигнатуры объемлющего пространства.

Эллиптические вложения, сигнатура $\{\sigma, \sigma, \zeta,-1,-1,-1\}$ (здесь и далее $\zeta, \sigma, \rho=$ $\pm 1)$ :

$$
\begin{gathered}
y^{0}=f(r) \sin (\alpha t+w(r)), \quad y^{1}=f(r) \cos (\alpha t+w(r)), \quad y^{2}=h(r), \\
y^{3}=r \cos \theta, \quad y^{4}=r \sin \theta \cos \varphi, \quad y^{5}=r \sin \theta \sin \varphi
\end{gathered}
$$

Параболические вложения, сигнатура $\{\zeta,-\zeta,-\zeta,-1,-1,-1\}$ :

$$
y^{+}=\frac{\alpha^{2} t^{2}}{2} h(r)+\alpha f(r) t+w(r), \quad y^{2}=f(r)+\alpha h(r) t, \quad y^{-}=h(r)
$$

(в этом и последующих вложениях, если не приведены выражения для компонент $y^{3}, y^{4}, y^{5}$, то они совпадают с приведенными в формуле (4)).

Гиперболические вложения, сигнатура $\{1,-1, \zeta,-1,-1,-1\}$ :

$$
y^{0}=f(r) \operatorname{sh}(\alpha t+w(r)), \quad y^{1}=f(r) \operatorname{ch}(\alpha t+w(r)), \quad y^{2}=h(r)
$$

или, при других значениях $r$,

$$
y^{0}=f(r) \operatorname{ch}(\alpha t+w(r)), \quad y^{1}=f(r) \operatorname{sh}(\alpha t+w(r)), \quad 0 y^{2}=h(r) .
$$

При работе с вложениями этого типа будем использовать функцию $\xi(r)$, которую положим равной 1 , если при данном $r$ применяется формула (5), и -1 , если применяется формула (7).

Спиральные вложения, сигнатура $\{\sigma, \sigma, \zeta,-1,-1,-1\}$ :

$$
y^{0}=f(r) \sin (\alpha t+w(r)), \quad y^{1}=f(r) \cos (\alpha t+w(r)), \quad y^{2}=k t+h(r) .
$$

Экспоненциальные вложения, сигнатура $\{1,-1, \zeta,-1,-1,-1\}$ :

$$
y^{+}=\frac{1}{\alpha} e^{\alpha t+w(r)}, \quad y^{-}=f(r) e^{-(\alpha t+w(r))}, \quad y^{2}=k t+h(r),
$$

где $y^{ \pm}=\left(y^{0} \pm y^{1}\right) / \sqrt{2}$, сигнатура указывается для лоренцевых компонент $y^{0}, y^{1}, \ldots$

Кубические вложения, сигнатура $\{\zeta,-\zeta,-\zeta,-1,-1,-1\}$ :

$$
y^{+}=\frac{\alpha^{2} \widetilde{t}^{3}}{6}+\alpha f(r) \widetilde{t}+u(r), \quad y^{2}=\frac{\alpha \widetilde{t}^{2}}{2}+f(r), \quad y^{-}=\widetilde{t},
$$

где $\tilde{t}=t+h(r)$. 



Рис. 1. Графики функций $g_{00}(r)$ для рассматриваемых черных дыр.

Кроме уже перечисленных шести вложений, еще есть три типа вложений, обладающих необходимой симметрией, однако они содержат слишком мало произвола для их использования при поиске вложений конкретных метрик. Для полноты картины выпишем их тоже: вложение с сигнатурой $\{\zeta, \sigma, \rho,-1,-1,-1\}$

$$
y^{0}=k_{0} t+h_{0}(r), \quad y^{1}=k_{1} t+h_{1}(r), \quad y^{2}=k_{2} t+h_{2}(r),
$$

вложение с сигнатурой $\{+1,+1,+1,-1,-1,-1\}$

$$
\begin{array}{ll}
y^{0}=r \operatorname{sh}(\alpha t+w(r)) \cos \theta, & y^{3}=r \operatorname{ch}(\alpha t+w(r)) \cos \theta, \\
y^{1}=r \operatorname{sh}(\alpha t+w(r)) \sin \theta \cos \varphi, & y^{4}=r \operatorname{ch}(\alpha t+w(r)) \sin \theta \cos \varphi, \\
y^{2}=r \operatorname{sh}(\alpha t+w(r)) \sin \theta \sin \varphi, & y^{5}=r \operatorname{ch}(\alpha t+w(r)) \sin \theta \sin \varphi
\end{array}
$$

и аналогичное (12) вложение с сигнатурой $\{-1,-1,-1,-1,-1,-1\}$, в котором sh и ch заменены на $\sin$ и $\cos$ (это вложение не годится для наших целей из-за евклидовости сигнатуры). В приведенных формулах $\alpha, k$ - положительные константы, а $f(r)$, $w(r), h(r)$ - произвольные функции.

Далее мы будем анализировать возможность построения глобальных вложений (4)-(12) для перечисленных выше метрик.

\section{3. АНАЛИЗ СУЩЕСТВОВАНИЯ ГЛОБАЛЬНЫХ ВЛОЖЕНИЙ}

Исследуем возможность построения глобальных (т. е. гладких при любых значениях $r$ ) вложений перечисленных выше типов для невращающихся черных дыр. Некоторые из перечисленных типов вложений не пригодны для этой цели, поскольку компонента $g_{00}$ метрики, индуцируемой вложениями этих типов, не может менять знак, в то время как все интересующие нас метрики обладают хотя бы одним горизонтом, в котором знак этой компоненты меняется (см. рис. 1).

Знак компоненты $g_{00}$ оказывается постоянным для вложений эллиптического типа, для которых подстановка (4) в (1) дает $g_{00}=\sigma \alpha^{2} f(r)^{2}$, параболического типа, где $g_{00}=-\zeta \alpha^{2} h(r)^{2}$, а также типа (11), где $g_{00}=\zeta k_{0}^{2}+\sigma k_{1}^{2}+\rho k_{2}^{2}=$ const, и типа (12), где $g_{00}=\alpha^{2} r^{2}$. Далее будем исследовать оставшиеся четыре типа вложений, а именно вложения (6)-(10). 
3.1. Гиперболические вложения. Будем учитывать, что для гиперболических вложений, задаваемых формулами (6), (7), выбор одной из этих двух формул определяется знаковой функцией $\xi(r)$. Используя $(1)$, вычисляем компоненты метрики

$$
g_{00}=\xi \alpha^{2} f^{2}, \quad g_{01}=\xi \alpha f^{2} w^{\prime}, \quad g_{11}=-\xi f^{\prime 2}+\xi f^{2} w^{\prime 2}+\zeta h^{\prime 2}-1 .
$$

Отсюда, используя (2) и (3), заключаем, что

$$
\xi=\operatorname{sign}(F), \quad f=\frac{\sqrt{|F|}}{\alpha}, \quad w^{\prime}=0,
$$

а также получаем возникающее из формулы для $g_{11}$ уравнение

$$
\zeta h^{\prime 2}=\frac{1}{4 \alpha^{2} F}\left(F^{\prime 2}-4 \alpha^{2}\right)+1 .
$$

В силу того что в некоторой точке $r_{*}$ функция $F(r)$ обращается в нуль, а $h(r)$ должна быть гладкой для искомого глобального вложения (поскольку она совпадает с компонентой $y^{2}$ функции вложения), из уравнения (15) следует, что $F^{\prime}\left(r_{*}\right)= \pm 2 \alpha$. Это означает, что либо функция $F(r)$ обращается в нуль только в одной точке, либо во всех точках, где это происходит, она имеет одинаковый модуль производной. Как видно из (3), последнее не происходит, если параметры черных дыр находятся в ситуации общего положения. Поэтому возможность построить глобальное гиперболическое вложение может существовать только для черных дыр S и S-AdS (см. табл. 1). Для метрики Шварцшильда такое вложение известно, это вложение Фронсдала [5]. Отметим, что прохождение функции $F(r)$ через нуль с изменением ее знака не приводит к нарушению гладкости функции вложения на горизонте, хотя $f(r)$ и $\xi(r)$ не являются гладкими (подробнее см. [5], [23]).

Исследуем случай S-AdS. Из уравнения (15), учитывая асимптотики его правой части, можно найти, что

$$
\zeta h^{\prime 2} \underset{r \rightarrow 0}{\longrightarrow}-\frac{m}{2 \alpha^{2} r^{3}}<0, \quad \zeta h^{\prime 2} \underset{r \rightarrow \infty}{\longrightarrow} \frac{|\Lambda|}{3 \alpha^{2}}+1>0 .
$$

Из второй асимптотики следует, что $\zeta=+1$, но это несовместимо с первой асимптотикой. Таким образом, глобальное гиперболическое вложение для черной дыры S-AdS построить не удается.

В результате получаем, что минимальное гиперболическое глобальное вложение существует только для черной дыры Шварцшильда.

3.2. Спиральные вложения. Используя (1), вычисляем компоненты метрики

$$
g_{00}=\sigma \alpha^{2} f^{2}+\zeta k^{2}, \quad g_{01}=\sigma \alpha f^{2} w^{\prime}+\zeta k h^{\prime}, \quad g_{11}=\sigma f^{\prime 2}+\sigma f^{2} w^{\prime 2}+\zeta h^{\prime 2}-1
$$

откуда, снова используя (2) и (3), заключаем, что

$$
\sigma=\operatorname{sign}\left(F-\zeta k^{2}\right), \quad f=\frac{\sqrt{\left|F-\zeta k^{2}\right|}}{\alpha}, \quad w^{\prime}=-\frac{\zeta \alpha k h^{\prime}}{F-\zeta k^{2}} .
$$

Поскольку $\sigma$ и $\zeta$ являются константами, отсюда следует, что $F(r)$ должно быть ограничено либо сверху, либо снизу, чтобы величина $F-\zeta k^{2}$ имела постоянный знак. Черные дыры S-AdS и RN-dS этим свойством не обладают, поэтому для них глобальное вложение построить не удается. Для черных дыр S, S-dS и RN глобальные минимальные вложения построены в работах [23], [28] и [26] соответственно. 
Остается только проанализировать возможность построения такого вложения для случая RN-AdS, что мы и сделаем.

Вид функции $F(r)$ для случая RN-AdS показывает, что для обеспечения знакоопределенности величины $F-\zeta k^{2}$ необходимо брать $\zeta=-1$ и $k^{2} \geqslant\left|F_{\min }\right|$, где $F_{\min }<0$ - минимум функции $F(r)$. Заметим, что если взять $k^{2}=\left|F_{\min }\right|$, то возникающее вложение содержит особенность в точке, где $F(r)=F_{\min }$, поэтому будем считать, что $k^{2}>\left|F_{\min }\right|$. Из формулы (1) для компоненты $g_{11}$ можно получить уравнение (в нем уже положено $\zeta=-1$ )

$$
h^{\prime 2}=\frac{F F^{\prime 2}-4 \alpha^{2}\left(F+k^{2}\right)(F-1)}{4 \alpha^{2} F^{2}} .
$$

Входящая в это уравнение функция $h(r)$, в отличие от входящей в уравнение (15), стремится к бесконечности на горизонтах из-за обращения в нуль стоящей в знаменателе функции $F(r)$. Это не обязательно означает отсутствие гладкости функции вложения на горизонтах, поскольку в компоненту функции вложения (8) функция $h(r)$ входит в виде суммы с $k t$, а при использовании времени внешнего наблюдателя $t$ имеется координатная сингулярность метрики - падение на горизонт происходит только за бесконечное время $t$.

Проверить гладкость функции вложения можно, перейдя к запаздывающему времени $\widetilde{t}=t+h(r) / k$ (в терминах которого $\left.y^{2}=k \widetilde{t}\right)$. При его использовании в (8) гладкость компоненты $y^{0}$ означает гладкость функции $u(r)=w(r)-\alpha h(r) / k$. Используя (18), в терминах $u(r)$ уравнение (19) можно переписать в виде

$$
u^{\prime 2}=\frac{F F^{\prime 2}-4 \alpha^{2}\left(F+k^{2}\right)(F-1)}{4 k^{2}\left(F+k^{2}\right)^{2}} .
$$

Поскольку в этом уравнении знаменатель нигде не обращается в нуль, функция вложения будет гладкой, если правая часть уравнения неотрицательна при всех $r$. Чтобы это проверить, прежде всего найдем ее асимптотику. Для функции $F(r)$, соответствующей случаю RN-AdS, находим

$$
u^{\prime 2} \underset{r \rightarrow 0}{\longrightarrow} \frac{q^{2}}{k^{2} r^{4}}>0, \quad u^{\prime 2} \underset{r \rightarrow \infty}{\longrightarrow} \frac{|\Lambda|-3 \alpha^{2}}{3 k^{2}} .
$$

Отсюда следует необходимое, но не достаточное ограничение на параметр $\alpha$, а именно: $\alpha^{2} \leqslant|\Lambda| / 3$. Поиск достаточного условия в данном случае оказывается весьма сложной задачей, и на данный момент она не решена. Поэтому вопрос о существовании глобального спирального вложения для черной дыры RN-AdS остается открытым.

В результате заключаем, что минимальные глобальные спиральные вложения существуют для черных дыр S, S-dS, RN и, возможно, RN-AdS.

3.3. Экспоненциальные вложения. Используя (1), вычисляем компоненты метрики

$$
g_{00}=-2 \alpha f+\zeta k^{2}, \quad g_{01}=f^{\prime}-2 f w^{\prime}+\zeta k h^{\prime}, \quad g_{11}=\frac{2}{\alpha} w^{\prime}\left(f^{\prime}-f w^{\prime}\right)+\zeta h^{\prime 2}-1
$$

откуда, используя (2) и (3), заключаем, что

$$
f=-\frac{F-\zeta k^{2}}{2 \alpha}, \quad w^{\prime}=\frac{F^{\prime}-2 \zeta \alpha k h^{\prime}}{2\left(F-\zeta k^{2}\right)} .
$$


Как и в случае спиральных вложений, проверить гладкость в терминах функций $h(r), w(r)$ нельзя (см. замечание после (19)). Поэтому уравнение, возникающее из формулы (1) для компоненты $g_{11}$, удобно записать в терминах функции

$$
u(r)=w(r)-\frac{\alpha}{k} h(r),
$$

которая должна быть гладкой для гладких вложений. Запишем сразу решение этого квадратного уравнения относительно $u^{\prime}(r)$ :

$$
u^{\prime}=\frac{k^{2} F^{\prime} \pm \sqrt{\zeta k^{2}\left(F F^{\prime 2}+4 \alpha^{2}\left(F-\zeta k^{2}\right)(F-1)\right)}}{2 k^{2}\left(F-\zeta k^{2}\right)} .
$$

Легко заметить, что если в некоторой точке $r_{*}$ функция $F(r)-\zeta k^{2}$ обращается в нуль, то стоящее в правой части (25) выражение будет гладким при определенном выборе знака перед корнем - этот знак должен быть противоположен знаку величины $F^{\prime}\left(r_{*}\right)$. Поскольку знак перед корнем должен выбираться единым образом для всех $r$, это означает, что вложение может быть всюду гладким только при таком выборе параметра $k$, при котором $F^{\prime}(r)$ имеет одинаковый знак во всех точках, где $F(r)=\zeta k^{2}$. Это условие будет использовано ниже.

Для гладкости также необходимо, чтобы подкоренное выражение (обозначим его через $P(r))$ в (25) было всюду неотрицательно. Поскольку для черных дыр $\mathrm{S}$ и $\mathrm{RN}$ глобальные минимальные вложения построены в работах [23] и [26] соответственно, проанализируем существование таких вложений для оставшихся четырех случаев. Запишем для них асимптотики подкоренного выражения (25). При $r \rightarrow 0$ имеем

S-dS, S-AdS: $\quad P(r) \underset{r \rightarrow 0}{\longrightarrow}-8 \zeta \frac{k^{2} m^{3}}{r^{5}}, \quad$ RN-dS, RN-AdS: $\quad P(r) \underset{r \rightarrow 0}{\longrightarrow} 4 \zeta \frac{k^{2} q^{6}}{r^{8}}$,

а при $r \rightarrow \infty$ для всех четырех случаев получаем

$$
P(r) \underset{r \rightarrow \infty}{\longrightarrow} \frac{4}{27} \zeta k^{2} \Lambda^{2}\left(3 \alpha^{2}-\Lambda\right) r^{4}+\frac{4}{9} k^{2} \Lambda\left(3 \alpha^{2} k^{2}-\zeta\left(3 \alpha^{2}-\Lambda\right)\right) r^{2}+\cdots
$$

(напомним, что $\Lambda>0$ для случаев $\mathrm{dS}$ и $\Lambda<0$ для случаев $\mathrm{AdS}$ ). Сравнение формул (24) и (25) показывает, что для черной дыры S-AdS ни при каком выборе $\zeta$ не удается обеспечить неотрицательность подкоренного выражения, а значит, глобальное вложение построить нельзя. Для случая S-dS необходимо брать $\zeta=-1$ и $\alpha \leqslant \sqrt{\Lambda / 3}$, а для RN-dS и RN-AdS $-\zeta=+1$, причем для RN-dS должно быть $\alpha \geqslant \sqrt{\Lambda / 3}$. Для случая S-dS при $\zeta=-1$ и для случая RN-AdS при $\zeta=+1$ можно заметить, что функция $F(r)-\zeta k^{2}$ будет обращаться в нуль обязательно дважды, причем в точках, где $F^{\prime}(r)$ имеет разные знаки (см. рис. 1$)$, поэтому для этих случаев глобального вложения построить нельзя (см. замечание после формулы (25)).

В результате остается возможность построения глобального вложения только для случая RN-dS при $\zeta=+1$ и $\alpha \geqslant \sqrt{\Lambda / 3}$. Если дополнительно положить $k=1$, то исследуемое подкоренное выражение примет вид

$$
P(r)=F F^{2}+4 \alpha^{2}(F-1)^{2} .
$$

Для соответствующей случаю RN-dS функции $F(r)$ (см. (3)) можно выбрать такое достаточно большое значение $\alpha$, что выражение (28) будет положительно при любых $r$ (хотя дать точную оценку для требуемого значения $\alpha$ является нетривиальной задачей). Следовательно, можно предъявить минимальное глобальное экспоненциальное вложение черной дыры RN-dS в виде

$$
y^{+}=\frac{1}{\alpha} e^{\alpha \widetilde{t}+u(r)}, \quad y^{-}=\frac{1-F(r)}{2 \alpha} e^{-(\alpha \widetilde{t}+u(r))}, \quad y^{2}=\widetilde{t}
$$


(остальные компоненты $y^{3,4,5}$ те же, что в (4)), где

$$
u(r)=\int d r \frac{F^{\prime}+\sqrt{F F^{\prime 2}+4 \alpha^{2}(F-1)^{2}}}{2(F-1)},
$$

$\widetilde{t}=t+h(r)$, а $h(r)$ имеет вид интеграла, который несложно записать с помощью формул (23), (24) и (30).

В результате заключаем, что минимальные глобальные экспоненциальные вложения существуют для черных дыр S, RN и RN-dS.

3.4. Кубические вложения. Используя (1), вычисляем компоненты метрики

$$
g_{00}=2 \zeta \alpha f, \quad g_{01}=\zeta\left(2 \alpha f h^{\prime}+u^{\prime}\right), \quad g_{11}=\zeta\left(2 \alpha f h^{\prime 2}-f^{\prime 2}+2 h^{\prime} u^{\prime}\right)-1,
$$

откуда, используя (2) и $(3)$, заключаем, что

$$
f=\zeta \frac{F}{2 \alpha}, \quad h^{\prime}=-\zeta \frac{u^{\prime}}{F} .
$$

Как видно из (10), для гладкого вложения гладкой должна быть функция $u(r)$, поэтому уравнение, возникающее из формулы (1) для компоненты $g_{11}$, удобно записать в терминах именно этой функции. Такое уравнение имеет вид

$$
u^{\prime 2}=1-F-\zeta \frac{F F^{\prime 2}}{4 \alpha^{2}} .
$$

Для гладкости функции $u(r)$, а значит, и всего вложения, необходима неотрицательность правой части этого уравнения при любых $r$. Для черных дыр $\mathrm{S}, \mathrm{S}-\mathrm{dS}$ и RN минимальные глобальные вложения построены в работах [23], [28] и [26] соответственно. Остается исследовать только возможность построения таких вложений для оставшихся трех случаев. Запишем для них асимптотики правой части (33). При $r \rightarrow 0$ имеем

$$
\text { S-AdS: } \quad u^{\prime 2} \underset{r \rightarrow 0}{\longrightarrow} 2 \zeta \frac{m^{3}}{\alpha^{2} r^{5}}, \quad \text { RN-dS, RN-AdS: } \quad u^{\prime 2} \underset{r \rightarrow 0}{\longrightarrow}-\zeta \frac{q^{6}}{\alpha^{2} r^{8}},
$$

а при $r \rightarrow \infty$ для всех трех случаев получаем

$$
u^{\prime 2} \underset{r \rightarrow \infty}{\longrightarrow} \zeta \frac{\Lambda^{3} r^{4}}{27 \alpha^{2}}
$$

Сравнивая эти формулы, легко заметить, что для черных дыр S-AdS и RN-dS ни при каком выборе $\zeta$ не удается обеспечить неотрицательность правой части (31), что означает невозможность построения глобальных вложений. Для оставшегося случая RN-AdS такая неотрицательность в пределах $r \rightarrow 0$ и $r \rightarrow \infty$ имеет место при $\zeta=-1$. Однако пока остается не ясным, можно ли выбором параметра $\alpha$ обеспечить ее при всех $r$, этот вопрос требует дополнительного исследования.

В результате заключаем, что минимальные глобальные кубические вложения существуют для черных дыр S, S-dS, RN и, возможно, RN-AdS.

\section{4. ЗАКЛЮЧЕНИЕ}

Результаты проведенного в настоящей работе анализа существования глобальных минимальных вложений невращающихся черных дыр собраны в табл. 2 (для типов вложения, не приведенных в табл. 2, глобальные вложения отсутствуют, см. начало раздела 3). Под глобальностью понимается гладкость вложения при всех значениях радиуса, а под минимальностью - минимально возможная размерность объемлющего пространства, в данном случае равная 6. В табл. 2 показана сигнатура 
объемлющего пространства для каждого известного вложения (число времениподобных направлений плюс число пространственноподобных), а также ссылка на работу, в которой это вложение было впервые построено (если ссылка не указана, оно построено в настоящей работе). Прочерком отмечены случаи, в которых соответствующее вложение не существует, а знаком вопроса - те случаи, для которых вопрос о существовании вложения пока остается открытым.

ТАБлицА 2. Глобальные минимальные вложения невращающихся черных дыр. Цифры показывают размерность и сигнатуру объемлющего пространства.

\begin{tabular}{|c|c|c|c|c|c|c|}
\hline Вложение & $\mathrm{S}$ & $\mathrm{S}-\mathrm{dS}$ & $\mathrm{S}-\mathrm{AdS}$ & $\mathrm{RN}$ & $\mathrm{RN}-\mathrm{dS}$ & $\mathrm{RN}-\mathrm{AdS}$ \\
\hline гиперболическое & $1+5,[5]$ & - & - & - & - & - \\
\hline спиральное & $1+5,[23]$ & $1+5,[28]$ & - & $2+4,[26]$ & - & $2+4, ?$ \\
\hline экспоненциальное & $1+5,[22]$ & - & - & $2+4,[26]$ & $2+4$ & - \\
\hline кубическое & $1+5,[23]$ & $1+5,[28]$ & - & $2+4,[26]$ & - & $2+4, ?$ \\
\hline
\end{tabular}

Для получения ответа на вопрос, существуют ли все-таки вложения в этих последних случаях, необходимо исследовать, обращаются ли в нуль на определенных отрезках некоторые полиномы высоких степеней (входящие в правые части уравнений (20) и (33)). Для этого может оказаться полезным метод “полной классификации

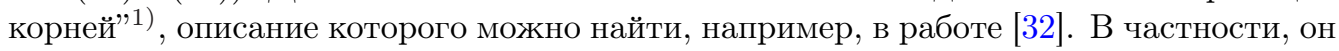
позволяет получить ограничения на коэффициенты параметрического многочлена, при которых он не имеет отрицательных корней. Этот метод успешно поддается алгоритмизации, однако требует значительных вычислительных мощностей.

Благодарности. Авторы благодарят организаторов конференции "В поисках фундаментальных симметрий”, посвященной 90-летию со дня рождения Ю. В. Новожилова, а также выражают свою благодарность А. Ерохину за предоставленные ссылки. Работа выполнена при поддержке Санкт-Петербургского государственного университета (грант № 11.38.223.2015).

\section{Список литературы}

[1] A. Friedman, J. Math. Mech., 10 (1961), 625-649.

[2] Д. Крамер, Х. Штефани, М. Мак-Каллум, Э. Херльт, Точные решения уравнений Эйнштейна, Энергоиздат, М., 1982.

[3] S. Willison, A Re-examination of the Isometric embedding approach to General Relativity, arXiv: 1311.6203.

[4] E. Kasner, Am. J. Math., 43:2 (1921), 130-133.

[5] C. Fronsdal, Phys. Rev., 116:3 (1959), 778-781.

[6] T. Regge, C. Teitelboim, "General relativity à la string: a progress report", Proceedings of the First Marcel Grossmann Meeting (Trieste, Italy, 1975), ed. R. Ruffini, North Holland, Amsterdam, 1977, 77-88.

[7] S. Deser, F. A. E. Pirani, D. C. Robinson, Phys. Rev. D, 14:12 (1976), 3301-3303.

[8] M. Pavsic, Phys. Lett. A, 107 (1985), 66-70.

[9] +V. Tapia, Class. Quantum Grav., 6:3 (1989), L49-L56.

[10] M. D. Maia, Class. Quantum Grav., 6:2 (1989), 173-183.

[11] I. A. Bandos, Modern Phys. Lett. A, 12:11 (1997), 799-810, arXiv: hep-th/9608093.

\footnotetext{
1) Complete root classification (CRC).
} 
[12] D. Karasik, A. Davidson, Phys. Rev. D, 67:6 (2003), 064012, 17 pp., arXiv: gr-qc/0207061.

[13] С. А. Пастон, В. А. Франке, ТМФ, 153:2 (2007), 271-288, arXiv: 0711.0576.

[14] Л. Д. Фаддеев, ТМФ, 166:3 (2011), 323-335, arXiv: 0911.0282; L. D. Faddeev, New action for the Hilbert-Einstein equations, arXiv: 0906.4639; 3+1 decomposition in the new action for the Einstein theory of gravitation, arXiv: 1003.2311.

[15] S. A. Paston, A. A. Sheykin, Internat. J. Modern Phys. D, 21:5 (2012), 1250043, 19 pp., arXiv: 1106.5212 .

[16] S. Deser, O. Levin, Phys. Rev. D, 59:6 (1999), 064004, 7 pp., arXiv: hep-th/9809159.

[17] S. A. Paston, JHEP, 06 (2014), 122, arXiv: 1402.3975.

[18] S. A. Paston, Class. Quantum Grav., 32:14 (2015), 145009, arXiv: 1411.4329.

[19] Y.-Z. Chu, J. Math. Phys., 55:9 (2014), 092501, 16 pp., arXiv: 1305.6933.

[20] K. R. Karmarkar, Proc. Indian Acad. Sci. A, 27:1 (1948), 56-60.

[21] T. Fujitani, M. Ikeda, M. Matsumoto, J. Math. Kyoto Univ., 1:1 (1961), 43-61.

[22] A. Davidson, U. Paz, Found. Phys., 30:5 (2000), 785-794.

[23] S. A. Paston, A. A. Sheykin, Class. Quantum Grav., 29:9 (2012), 095022, 17 pp., arXiv: 1202.1204.

[24] J. Rosen, Rev. Modern Phys., 37:1 (1965), 204-214.

[25] J. Rosen, Nuovo Cimento, 38 (1965), 631-633.

[26] S. A. Paston, A. A. Sheykin, SIGMA, 10 (2014), 003, 10 pp., arXiv: 1304.6550.

[27] Y.-W. Kim, Y.-J. Park, K.-S. Soh, Phys. Rev. D, 62:10 (2000), 104020, 6 pp., arXiv: gr-qc/0001045.

[28] A. A. Sheykin, D. A. Grad, S. A. Paston, PoS( QFTHEP2013), 091, arXiv: 1401.7820.

[29] Р. Р. Кузеев, Гравитация и теория относительности, 18 (1981), 75-82.

[30] Р. Р. Кузеев, Гравитация и теория относительности, 21 (1984), 123-126.

[31] S.-T. Hong, S.-W. Kim, J. Korean Phys. Soc., 49 (2006), S748-S754, arXiv: gr-qc/0503079.

[32] L. Songxin, J. J. David, J. Symbolic Comput., 44:10 (2009), 1487-1501. 